\title{
Regulation of cAMP intracellular levels in human platelets stimulated by 2-
}

arachidonoylglycerol

\section{Maria Grazia Signorello, Giuliana Leoncini*}

Department of Pharmacy, Biochemistry Lab, University of Genoa, Viale Benedetto XV 3, 16132 Genova, Italy,

Correspondence to: *Giuliana Leoncini, Dept. of Pharmacy, Biochemistry Lab, University of Genoa, Viale Benedetto XV 3, 16132 Genoa, Italy. Tel.: +39-0103538154; FAX: +39-0103538153; E-mail: leoncini@unige.it

Running Head: 2-arachidonoylglycerol effect on cAMP level

Bullet Point Keywords:

- 2-Arachidonoylglycerol;

- $\mathbf{C A M P}$;

- Phosphodiesterase 3A;

- Human platelets

Total number of table: 1

Total number of text figures: 7

Grant Information:

- Contract grant sponsor: Ministero della Ricerca Scientifica, Italy.

- Contract grant number: Cofin MIUR 2009 project n 02030200605

Abbreviations: 2-AG, 2-arachidonoylglycerol; MLC, myosin light chain; PDE, phosphodiesterase; 


\begin{abstract}
We demonstrated that in human platelets the endocannabinoid 2-arachidonoylglycerol (2-AG) decreased dose- and time-dependently cAMP intracellular levels. No effect on cAMP decrease induced by 2-AG was observed in the presence of the adenylate cyclase inhibitor SQ22536 as well in platelets pretreated with the thromboxane A2 receptor antagonist, SQ29548 or with aspirin, inhibitor of arachidonic acid metabolism through the cyclooxygenase pathway. An almost complete recovering of cAMP level was measured in platelets pretreated with the specific inhibitor of phosphodiesterase (PDE) 3A, milrinone. In platelets pretreated with LY294002 or MK2206, inhibitors of PI3K/AKT pathway, and with U73122, inhibitor of phospholipase C pathway, only a partial prevention was shown. cAMP intracellular level depends on synthesis by adenylate cyclase and hydrolysis by PDEs. In 2-AG-stimulated platelets adenylate cyclase activity seems to be unchanged. In contrast PDEs appear to be involved. In particular PDE3A was specifically activated, as milrinone reversed cAMP reduction by 2-AG. 2-AG enhanced PDE3A activity through its phosphorylation. The PI3K/AKT pathway and PKC participate to this PDE3A phosphorylation/activation mechanism as it was greatly inhibited by platelet pretreatment with LY294002, MK2206, U73122 or the PKC specific inhibitor GF109203X. Taken together these data suggest that 2-AG potentiates its power of platelet agonist reducing cAMP intracellular level.
\end{abstract}


Endogenous cannabinoids are biologically active lipids that include $\mathrm{N}$-arachidonoylethanolamine (anandamide) and 2arachidonoylglycerol (2-AG) [Petrosino et al., 2009]. Anandamide and 2-AG are the main endogenous agonists of type1 (CB1) and type-2 (CB2) cannabinoid receptors [Salzet et al., 2000], both of which belong to the superfamily of Gprotein-coupled receptors. Apparently, 2-AG is the true physiological agonist of CB1 and CB2 [Sugiura and Waku, 2002], whereas anandamide might be the ligand of CB1 and other targets like vanilloid receptors [Petrosino et al., 2009]. 2-AG can be formed from membrane phospholipids or lysophosphatidic acid, and elicits a variety of biological responses "in vitro" and "in vivo" by targeting CB receptors in an autocrine and/or paracrine manner [Sugiura et al., 2006]. For instance, 2-AG induces calcium transients [Sugiura and Waku, 2002] and activates various kinases such as p42/44 mitogen-activated protein kinase (MAPK) [Kobayashi et al., 2001] and p38MAPK [Derkinderen et al., 2001]. Additionally, 2-AG behaves as a retrograde messenger in synaptic transmission [Sugiura et al., 1997], and it regulates various types of inflammatory and immune responses, spanning from increased production of chemokines [Kishimoto et al., 2004] to induction of migration of macrophage-like cells [Kishimoto et al., 2003] and B-lymphocytes [Jordà et al., 2002]. Remarkably, endocannabinoids play major roles within the cardiovascular system [Randall, 2007; Mach et al., 2009]. Human vascular endothelial cells and human platelets generate 2-AG upon stimulation [Sugiura et al., 1988; Maccarrone et al., 2001]. Human platelets have the biochemical tools to metabolize endocannabinoids [Maccarrone et al., 2001], express authentic CB1 and CB2 receptors [Catani et al., 2010], and are activated by micromolar concentrations of 2-AG through a CB1/CB2-dependent mechanism that leads to increased intracellular calcium and inositol-1,4,5 trisphosphate, and decreased cAMP [Maccarrone et al., 2001]. In contrast Baldassarri et al. [2008] have published that the ability of 2-AG to act as a physiological agonist does not need CB1/CB2 receptor, leaving open the question of which molecular mechanism underlies the effect of this endocannabinoid. Platelet treatment with 2-AG leads to the p38MAPK/cytosolic phospholipase $\mathrm{A}_{2}$ pathway activation [Signorello et al., 2011a] and to NO/cGMP pathway regulation by PKC [Signorello et al., 2011b]. Moreover 2-AG induces a rapid myosin light chain (MLC) phosporylation/activation, early mediated by Rho kinase and later by MLC kinase. MLC phosphorylation is potentiated by the MLC phosphatase inhibition through the phosphorylation of both MYPT1 and CPI-17 subunits [Signorello et al., 2013]. Recently it has been shown that the PI3K/AKT pathway is involved in MLC phosphorylation and some downstream events such as actin polymerization, ATP secretion and aggregation [Signorello and Leoncini, 2014]. In addition an ultrastructural study [Malorni et al., 2004] put in evidence a significant increase of F-actin in 2-AG treated platelets.

Cyclic AMP is a control molecule in platelets that interrupts multiple signalling pathways and plays a significant role in down-regulation of platelet activation [Smolenski, 2012; Raslan and Naseem, 2014]. Starting from a previous report by Maccarrone et al. [2001] we wanted to investigate the mechanisms involved in cAMP reduction by 2-AG in 
stimulated human platelets. The levels of cAMP are tightly controlled and are dependent on its rate of synthesis by adenylate cyclase and its rate of hydrolysis by cAMP phosphodiesterases (PDEs). Previous observations have shown that platelet activation is associated with a decrease in cAMP concentration [Maccarrone et al., 2001; Lagarde and Dechavanne, 1977]. More recently Hunter et al. [2009] demonstrated that in platelets stimulated by several agonists, including thrombin, cAMP intracellular levels are reduced. The PKC-mediated phosphorylation/activation of PDE3A appear to be involved in this mechanism. On the other hand Zang and Colman [2007] have shown that the activation of PDE3A is AKT-mediated and is involved in regulation of cAMP during platelet activation by thrombin.

Therefore we wanted to clarify whether 2-AG was able to potentiate its power of platelet agonist decreasing cAMP intracellular levels. In the present study we show that 2-AG is able to reduce cAMP intracellular levels through the activation of cAMP-dependent PDE3A. 2-AG raises PDE3A activity by promoting its phosphorylation. The PI3K/AKT signalling pathway is responsible, at least in part, for PDE3A activation. In addition PKC seems to be partially involved in the mechanisms leading to PDE3A activition.

\section{MATERIALS AND METHODS}

\section{Materials}

Apyrase, aspirin (ASA), Colorburst ${ }^{\mathrm{TM}}$ electrophoresis markers, cilostamide, cilostazol, dithiothreithol (DTT), EHNA, forskolin, GF109203X, isobutylmethilxanthine (IBMX), leupeptin, $\square \beta$-mercaptoethanol, milrinone, prostaglandin $\mathrm{E}_{1}$ (PGE 1 ), PMSF, protease inhibitor cocktail (product number: P2714), U73122 and all chemicals were from SigmaAldrich, USA. 2-AG, LY294002 and SQ22536 were from Tocris Bioscience, UK. MK2206 was from Selleck Chem and SQ29548 from Cayman Chem, USA. Inhibitors were diluted in saline from a stock DMSO solution immediately before each experiment. Cyclic AMP EIA kit (catalog $\mathrm{N}^{\circ}$ 900-163) and cyclic nucleotide phosphodiesterase assay kit (catalog $\mathrm{N}^{\circ}$ BML-AK800) were from Enzo Life Sciences, USA and adenylate cyclase assay kit (catalog N ${ }^{\circ}$ GQE-ELH0268) was from Elab Sciences, China. Anti-PDE3A (catalog $\mathrm{N}^{\circ}$ 07-2197) and anti-phosphoserine (catalog $\mathrm{N}^{\circ}$ AB1603) were obtained by Merck-Millipore USA. Anti-phospho-PDE (ser290/ser293) (catalog N MBS540101) was from Biosource, USA. Protein A-sepharose was obtained by GE Healthcare, USA. Horseradish peroxidase-conjugated secondary antibodies and $\beta$-actin were purchased from Santa Cruz Biotechnology, USA. Nitrocellulose membranes (pore size $0.45 \mu \mathrm{m}$ ) were from Bio-Rad Laboratories, USA.

\section{Blood collection and preparative procedures}

Freshly drawn venous blood from healthy volunteers of the "Centro Trasfusionale, Ospedale San Martino" in Genoa was collected into $130 \mathrm{mM}$ aqueous trisodium citrate anticoagulant solution (9:1). The donors claimed to have not taken drugs known to interfere with platelet function during two weeks prior to blood collection, and gave their informed 
consent. Washed platelets were prepared centrifuging whole blood at $100 \times \mathrm{g}$ for $25 \mathrm{~min}$. To the obtained platelet-rich plasma (PRP) $4 \mathrm{mU} / \mathrm{mL}$ apyrase and $4 \mu \mathrm{M} \mathrm{PGE}_{1}$ were added. PRP was then centrifuged at $1100 \times \mathrm{g}$ for $15 \mathrm{~min}$. Pellet was washed once with $\mathrm{pH} 5.2 \mathrm{ACD}$ solution (75 mM trisodium citrate, $42 \mathrm{mM}$ citric acid and $136 \mathrm{mM}$ glucose), centrifuged at $1100 \times \mathrm{g}$ for $15 \mathrm{~min}$ and then resuspended in calcium-free $10 \mathrm{mM}$ HEPES buffer containing $145 \mathrm{mM}$ $\mathrm{NaCl}, 5 \mathrm{mM} \mathrm{KCl}, 1 \mathrm{mM} \mathrm{MgSO} 4,10 \mathrm{mM}$ glucose (pH 7.4).

\section{cAMP measurement assay}

Washed platelets $\left(1.0 \times 10^{9}\right.$ platelets $\left./ \mathrm{mL}\right)$, preincubated for $10 \mathrm{~min}$ at $37^{\circ} \mathrm{C}$ with saline, were stimulated with 2 -AG. In the experiments in which the dose-dependent effect of the endocannabinoid was evaluated, platelets were stimulated with increasing concentrations of 2-AG for $120 \mathrm{sec}$. In the experiments in which the time-dependence was assessed, platelets were challenged with $10 \mu \mathrm{M} 2-\mathrm{AG}$ for different times. Moreover washed platelets $\left(1.0 \times 10^{9} \mathrm{platelets} / \mathrm{mL}\right)$, preincubated for $10 \mathrm{~min}$ at $37^{\circ} \mathrm{C}$ with varying compounds, were stimulated with $10 \mu \mathrm{M} 2-\mathrm{AG}$ for 120 sec. In all experiments reaction was stopped by adding cold $2.0 \mathrm{M}$ perchloric acid. Mixtures were then centrifuged for 3 min at $12000 \times \mathrm{g}$ and the supernatants, neutralised with $2.0 \mathrm{M} \mathrm{NaOH}$, were analysed for cAMP content by a commercial EIA kit, following the manufacturer's instructions with appropriate modifications.

\section{Adenylate cyclase activity assay}

Washed platelets $\left(1.0 \times 10^{9}\right.$ platelets $\left./ \mathrm{mL}\right)$, preincubated for $10 \mathrm{~min}$ at $37^{\circ} \mathrm{C}$ with saline, were stimulated with increasing concentration of $2-\mathrm{AG}$ for $120 \mathrm{sec}$. Washed platelets $\left(1.0 \times 10^{9}\right.$ platelets $\left./ \mathrm{mL}\right)$, preincubated for 10 min at $37^{\circ} \mathrm{C}$ with varying compounds, were stimulated with $10 \mu \mathrm{M} 2-\mathrm{AG}$ for $120 \mathrm{sec}$. In all experiments reaction was stopped by putting samples on ice. Samples, added with $10 \mu \mathrm{g} / \mathrm{mL}$ leupeptin, $1 \mathrm{mM}$ PMSF, $100 \mu \mathrm{M}$ DTT, were sonicated twice for $15 \mathrm{sec}$ and then centrifuged at $14000 \times \mathrm{g}$ for $5 \mathrm{~min}$. Adenylate cyclase activity was assayed in the supernatants by a commercial EIA kit, following the manufacturer's instructions with appropriate modifications.

\section{PDE activity assay}

Washed platelets $\left(1.0 \times 10^{9}\right.$ platelets $\left./ \mathrm{mL}\right)$, preincubated for $10 \mathrm{~min}$ at $37^{\circ} \mathrm{C}$ with saline, were stimulated with 2 -AG. In the experiments in which the dose-dependent effect of the endocannabinoid was evaluated, platelets were stimulated with increasing concentration of 2-AG for $120 \mathrm{sec}$. When the time-dependence was assessed, platelets were challenged with $10 \mu \mathrm{M} 2$-AG for different incubation times. On the other hand washed platelets $\left(1.0 \times 10^{9}\right.$ platelets $\left./ \mathrm{mL}\right)$, preincubated for $10 \mathrm{~min}$ at $37^{\circ} \mathrm{C}$ with varying compounds able to inhibit specific signal transduction pathways, were stimulated with $10 \mu \mathrm{M}$ 2-AG for $120 \mathrm{sec}$. In every experimental conditions, the reaction was stopped by putting 
samples on ice. To each samples $10 \mu \mathrm{g} / \mathrm{mL}$ leupeptin, $1 \mathrm{mM}$ PMSF, $100 \mu \mathrm{M}$ DTT were added. Platelet suspensions, sonicated twice for $15 \mathrm{sec}$, were centrifuged at $14000 \times \mathrm{g}$ for $5 \mathrm{~min}$. PDE activity was assayed in the supernatants by a commercial EIA kit using cAMP as substrate and following the manufacturer's instructions with appropriate modifications.

\section{Immunoprecipitation of proteins}

Washed platelets $\left(1.0 \times 10^{9}\right.$ platelets $\left./ \mathrm{mL}\right)$, preincubated for $10 \mathrm{~min}$ at $37^{\circ} \mathrm{C}$ with saline, were stimulated with 2 -AG. In the experiments in which the dose-dependent effect of the endocannabinoid was evaluated, platelets were stimulated with increasing concentration of 2-AG for $120 \mathrm{sec}$. When the time-dependence was assessed, platelets were challenged with $10 \mu \mathrm{M} 2-\mathrm{AG}$ for different incubation times. Washed platelets $\left(1.0 \times 10^{9}\right.$ platelets $\left./ \mathrm{mL}\right)$, preincubated for 10 min at $37^{\circ} \mathrm{C}$ with varying compounds able to inhibit several signal transduction pathways, were stimulated with $10 \mu \mathrm{M} 2-\mathrm{AG}$ for $120 \mathrm{sec}$. Incubation was stopped by adding to samples an equal volume of lysis mixture $(0.5 \%$ SDS, $1 \%$ Triton X100, 0.75\% sodium deoxycholate, $10 \mathrm{mM}$ EDTA, $1 \mathrm{mM}$ PMSF, $50 \mathrm{mM} \mathrm{NaF,} 200 \mu \mathrm{M} \mathrm{Na}_{3} \mathrm{VO}_{4}, 1 / 100$ dilution protease inhibitor cocktail). Lysates, after a brief centrifugation, were treated for $2 \mathrm{~h}$ at $4^{\circ} \mathrm{C}$ with $1.0 \mu \mathrm{g}$ of anti-PDE3A antibody. The immunocomplexes were precipitated with protein A-sepharose. After 60 min on ice, immunoprecipitates were washed with IP-wash 1 (10 mM pH 7.4 Tris/HCl, $150 \mathrm{mM} \mathrm{NaCl}, 0.5 \%$ Triton X-100), followed by IP-wash 2 (10 mM pH 7.Tris/HCl, $750 \mathrm{mM} \mathrm{NaCl}, 0.5 \%$ Triton X-100) and finally with IP-wash 1 . Immunoprecipitates were extracted with $2 \times$ Laemmli-SDS reducing sample buffer, heated at $80^{\circ} \mathrm{C}$ for $15 \mathrm{~min}$ and resolved on 5-10\% SDS-PAGE. Running was performed in the presence of Colorburst ${ }^{\mathrm{TM}}$ Electrophoresis weight markers. Gels were transferred to nitrocellulose membranes and blots were blocked in 5\% BSA dissolved in TBST (Tris buffer saline, pH 7.6, containing $10 \mathrm{mM}$ Tris, $150 \mathrm{mM} \mathrm{NaCl}$, and $0.1 \%$ Tween 20 ) at $37^{\circ} \mathrm{C}$ for $30 \mathrm{~min}$ and then incubated overnight at $4^{\circ} \mathrm{C}$ with anti-phosphoserine (1:500 dilution) antibody. Membranes were extensively washed and incubated for 60 min at room temperature with horseradish peroxidase-conjugated secondary antibody. After further washings, blots were developed using the ECL ${ }^{\circledR}$ system. Optical density, reported as arbitrary units, was directly quantified by the Bio-Rad Chemi-Doc software package. Nitrocellulose membranes were then stripped by incubation with $62.5 \mathrm{mM}$ Tris/HCl (pH 6.7), 2\% SDS, 100 $\mu \mathrm{M} \beta$-mercaptoethanol for $30 \mathrm{~min}$ at $50^{\circ} \mathrm{C}$, reprobed with anti-PDE3A and band intensity was quantified as detailed above.

\section{Immunoblotting analysis of phospho-proteins}

Washed platelets $\left(1.0 \times 10^{9}\right.$ platelets $\left./ \mathrm{mL}\right)$, preincubated for $10 \mathrm{~min}$ at $37^{\circ} \mathrm{C}$ with saline, were stimulated with 2 -AG. In the experiments in which the dose-dependent effect of the endocannabinoid was evaluated, platelets were stimulated 
with increasing concentration of 2-AG for $120 \mathrm{sec}$. When the time-dependence was assessed, platelets were challenged with $10 \mu \mathrm{M} 2$-AG for different incubation times. In other experiments platelets $\left(1.0 \times 10^{9}\right.$ platelets $\left./ \mathrm{mL}\right)$, preincubated for 10 min at $37^{\circ} \mathrm{C}$ with varying compounds able to inhibit several signal transduction pathways, were stimulated withy 10 MM 2-AG for $120 \mathrm{sec}$. Incubation was stopped by adding $2 \times$ Laemmli-SDS reducing sample buffer. Samples, heated for $5 \mathrm{~min}$ at $100^{\circ} \mathrm{C}$, were separated by $5-10 \%$ SDS-PAGE, and transferred to nitrocellulose membranes. Running was performed in the presence of Colorburst ${ }^{\mathrm{TM}}$ Electrophoresis weight markers. Blots were blocked in $5 \%$ BSA dissolved in TBST (Tris buffer saline, pH 7.6, containing $10 \mathrm{mM}$ Tris, $150 \mathrm{mM} \mathrm{NaCl}$, and $0.1 \%$ Tween 20) at $37^{\circ} \mathrm{C}$ for $30 \mathrm{~min}$, and then incubated overnight at $4^{\circ} \mathrm{C}$ with anti-phospho-PDE3A (ser290/ser293) (1:500 dilution) antibody. Membranes were extensively washed and incubated for $60 \mathrm{~min}$ at room temperature with horseradish peroxidase-conjugated secondary antibody. After further washings, blots were developed using the ECL ${ }^{\circledR}$ system, and the optical density, reported as arbitrary units, was directly quantified by the Bio-Rad Chemi-Doc software package. Nitrocellulose membranes were then stripped by incubation with $62.5 \mathrm{mM}$ Tris/ $\mathrm{HCl}$ (pH 6.7), $2 \% \mathrm{SDS}, 100 \mu \mathrm{M} \beta$-mercaptoethanol for $30 \mathrm{~min}$ at $50^{\circ} \mathrm{C}$, reprobed with anti- $\beta$-actin. Band intensity was quantified as detailed above.

\section{Platelet aggregation}

Platelet aggregation was performed in a Menarini Aggrecoder according to Born's method [Born, 1962] and quantified by the light transmission reached within $3 \mathrm{~min}$. Washed platelets $\left(3.0 \times 10^{8} \mathrm{plts} / \mathrm{mL}\right)$ were preincubated with saline or inhibitors for $2 \mathrm{~min}$ at $37^{\circ} \mathrm{C}$ before platelet stimulation with $10 \mu \mathrm{M}$ 2-AG. To calculate the percentage of inhibition, the extent of aggregation measured in the presence of the inhibiting agent was always compared with that measured in a control sample containing saline, carried out under the same conditions. From each series of experiments, in which the inhibitors were tested in at least five concentrations, a percentage inhibition-concentration curve was derived. From this curve, the IC50 value was calculated as the concentration of inhibitor causing a $50 \%$ inhibition of the aggregation.

\section{Statistical analysis}

Data are mean \pm SD of at least four independent experiments, each performed in duplicate. Statistical comparison between two groups was made by the unpaired Student's t-test. One-way ANOVA followed by Bonferroni's post hoc test was used to compare multiple groups. Statistical significance was defined as $\mathrm{p}<0.05$.

\section{RESULTS}

\section{Effect of 2-AG on cAMP level}

Intracellular cAMP level was decreased in platelets stimulated by 2-AG. The 2-AG effect was dose-dependent. Total cAMP level, $6.6 \pm 0.4 \mathrm{pmol} / 10^{8}$ plts in resting platelets, decreased to $4.1 \pm 0.2 \mathrm{pmol} / 10^{8} \mathrm{plts}$ in platelets stimulated with 10 
$\mu \mathrm{M}$ 2-AG (Fig. 1A). Moreover the time-dependent 2-AG effect was gradual, peaking after $120 \mathrm{sec}$ of platelet stimulation with 2-AG (Fig. 1B). In contrast 2-AG did not decrease cGMP level in human platelets, but it produced a light increase of this cyclic nucleotide. The basal cGMP concentration that corresponded to $0.6 \pm 0.1 \mathrm{pmol} / 10^{8} \mathrm{plts}$ in resting platelets increased to $0.8 \pm 0.1 \mathrm{pmol} / 10^{8}$ plts in platelets stimulated by $10 \mu \mathrm{M}$ 2-AG as previously observed [Signorello et al., 2011b]. A small increase in cGMP levels after exposure of platelets to von Willebrand factor [Riba et al., 2006; Gambaryan et al., 2008], thrombin [Zhang et al., 2011] or collagen [Riba et al., 2006] was described. On the other hand NO is increased in 2-AG-stimulated platelets [Signorello et al., 2011b]. NO activates soluble guanylyl cyclase resulting in stimulation of synthesis of cGMP. Thrombin, von Willebrand factor and collagen are also able to activate soluble guanylyl cyclase although to a lesser extent than NO. The 2-AG effect on cAMP level could be explained as suppression of cAMP synthesis by inhibiting adenylate cyclase or by increasing its hydrolysis by activation of cAMP PDE. No significant effect of 2-AG on adenylate cyclase activity was shown as the measured activity was $48 \pm 8$ and $41 \pm 4 \mathrm{pmol} \mathrm{cAMP} / \mathrm{min} / \mathrm{mg}$ protein in the absence or in the presence of $10 \mu \mathrm{M} 2$-AG, respectively

(Fig. 2), in agreement with previous data [Leoncini et al., 2004]. IBMX lightly stimulates adenylate cyclase activity, while forskolin induces a strong stimulation (Fig. 2). In order to clarify mechanisms involved in 2-AG effect, we tested the ability of 2-AG to reduce total intracellular cAMP level in the presence of varying inhibitors. We found that the cAMP decrease was unaffected in platelets preincubated with the adenylate cyclase inhibitor, SQ22536, or with the PDE2 inhibitor EHNA, whereas it was almost completely blocked in platelets pretreated with the PDE3A specific inhibitor milrinone, suggesting that PDE3A activation plays the major role in the reduction of the intracellular cAMP level (Fig. 1C). To clarify the mechanisms involved in the upstream signalling pathway of PDE3A activation, we tested the effect of LY294002 and MK2206 inhibitors of PI3K and AKT, respectively and of U73122, inhibitor of PLC $\gamma$ pathway. In the presence of LY294002 and MK2206 a significant (p<0.0005) recovery of cAMP was obtained. A lower effect was observed in platelets pretreated with U73122 (Fig. 1C). No experiments have been carried out in the presence of the specific PKC inhibitor GF109203X as this compound interferes with the cAMP assay. In addition in platelets pretreated with ASA or SQ29548, inhibitor of thromboxane receptor no 2-AG effect on cAMP intracellular level was demonstrated. Finally experiments in platelets not stimulated with 2-AG have been performed. Results were quite similar to those obtained in the presence of 2-AG (data not shown).

\section{Activation of PDE3A by 2-AG}

Data reported in Fig. 3 show that PDE is activated in platelets stimulated with 2-AG. The 2-AG effect was dosedependent peaking at $10 \mu \mathrm{M}$ (Fig. 3A). Moreover platelet PDE activity gradually increased during platelet incubation 
with 2-AG, reaching the highest activation after $120 \mathrm{sec}$. A prolonged incubation did not raise the 2-AG effect (Fig. $3 B)$.

Platelets express several PDEs: PDE2, PDE3 and PDE5. PDE2A, which hydrolyses both cGMP and cAMP with an high $\mathrm{Km}$, is allosterically stimulated by cGMP at moderate levels and may contrast the stimulated levels of cAMP [Colman, 2004]. PDE3A can hydrolyze both cAMP and cGMP with the same Km but at 10-fold higher Vmax for cAMP than cGMP, PDE5 which prefers cGMP as a substrate [Haslam et al., 1999; Smolenski, 2012]. To clarify which of these enzymes was stimulated by 2-AG we carried out experiments in the presence of several PDE inhibitors. When platelets were treated with the PDE3A inhibitor milrinone PDE activation induced by 2-AG was cancelled. The same result was obtained with cilostazol and cilostamide (data not shown). On the contrary 2-AG was still able to activate PDE in platelets pretreated with the PDE2 selective inhibitor EHNA (Fig. 3C), indicating that PDE3A but not PDE2 is involved in 2-AG activation of cAMP-PDE. In addition LY294002, MK2206 and U73122 produced a partial inhibition of 2-AG effect on PDE3A suggesting that PI3K/AKT and PLC/PKC pathways are both upstream involved in PDE3A activation (Fig. 3C). Finally SQ29548 or ASA did not modify the 2-AG effect on PDE3A activation, excluding that a 2AG metabolite such as arachidonic acid could participate to 2-AG effect.

\section{PDE3A phosphorylation upon platelet activation by 2-AG}

In platelets stimulated by 2-AG PDE3A is activated (Fig. 3). As it occurs in platelets activated by varying agonists such as thrombin [Hunter et al., 2009; Zhang and Coleman, 2007], PDE3A is co-regulated by phosphorylation. Thus by immunoprecipitation technique we have measured the phosphorylation status of PDE3A. We have shown that 2-AG induces PDE3A phosphorylation. The 2-AG effect is dose-dependent peaking at $10 \mu \mathrm{M}$ (Fig. 4A) and time-dependent reaching the maximum after $120 \mathrm{sec}$ of incubation (Fig. 4B). In addition we have tested the effect of several inhibitors on PDE3A phosphorylation (Fig. 5). We have found that the PDE2 inhibitor EHNA has no effect, while milrinone abolishes PDE3A phosphorylation. LY294002 and MK2206, PI3K/AKT inhibitors, U73122 and GF109203X, PLC $\gamma /$ PKC pathway inhibitors, significantly $(\mathrm{P}<0.0001)$ reduce PDE3A phosphorylation. On the contrary SQ29548 or ASA do not change the 2-AG effect. These results parallel those on PDE3A activity (Fig. 3C), suggesting that phosphorylation and activation are strictly connected. It is known that phosphorylation/activation of PDE3A could involve varying serine residues. In particular ser428 seems to be target of PKC and ser290/293 can be phosphorylated through the PI3K/AKT pathway activation [Hunter et al., 2009]. Results reported in Fig. 5 indicate that both PKC and PI3K/AKT pathway can participate to the PDE3A phosphorylation. Unfortunately we haven't the possibility to evaluate the phosphorylation status of ser428, as we did not dispose of the specific antibody against this residue. In contrast we have quantified the phosphorylation level of ser290/293 in response to 2-AG. We have found that the 2-AG effect on 
ser290/293 phosphorylation is dose- and time-dependent reaching the maximum level at $10 \mu \mathrm{M} 2$-AG and 120 sec of incubation with the endocannabinoid (Fig. 6). As expected the phosphorylation of this residue was cancelled by milrinone and greatly reduced by the inhibitors of PI3K/AKT pathway, LY294002 and MK2206 (Fig. 7).

\section{Effect of PDE3A inhibitors on platelet aggregation induced by 2-AG}

We have evaluated the effect of some known PDE3A inhibitors on 2-AG induced aggregation. We have found (Table 1) that cilostazol or cilostamide inhibit aggregation with a similar value of IC50 which corresponds to $4.2 \pm 0.7$ and $4.4 \pm 0.7$ $\mu \mathrm{M}$ while milrinone has showed an higher, but non significantly different value $(5.1 \pm 0.8 \mu \mathrm{M})$.

\section{DISCUSSION}

Results reported in literature [Maccarrone et al., 2001; Baldassarri et al., 2008; Signorello et al., 2011a; Signorello et al., 2011b; Signorello et al., 2013; Signorello and Leoncini, 2014; Malorni et al., 2004] suggest that 2-AG behaves as a true agonist of human platelets. Concerning the mechanism by which 2-AG acts in human platelets to date independent investigations have provided conflicting results, that span from evidence for a CB1/CB2-dependent mechanism to a non CB1/CB2-dependent signal transduction or to a classical arachidonate-dependent pathway. Previous data suggest that 2AG induced aggregation is cannabinoid receptor dependent and metabolic breakdown independent [Maccarrone et al., 2001], in agreement with more recent data indicating that 2-AG does not activate human platelets through the release of arachidonic acid [Signorello et al., 2011a]. In contrast data presented by Kweon et al [2010] and Brantl et al [2014] have shown that 2-AG stimulates platelets by a MAGL (monoacylglycerol lipase) triggered mechanism leading to free arachidonic acid and its metabolism. CB1, CB2 or non CB1/CB2 seems to be not involved, as suggested by Baldassari et al [2008]. On the contrary we have shown that 2-AG activates the p38MAPK/cPLA 2 pathway [Signorello et al., 2011a] and promotes myosin light chain phosphorylation [Signorello et al., 2013] through a CB1 receptor mechanism. In the present report we show that 2-AG potentiates its power of agonist reducing intracellular cAMP level. It is known that ADP reduces cAMP concentration below the basal levels through the ADP secreted from granules during platelet activation. Two different $\mathrm{P} 2$ receptors, $\mathrm{P} 2 \mathrm{Y}_{1}$ and $\mathrm{P}_{2} \mathrm{Y}_{12}$, involved in the ADP-induced platelet responses have been cloned. The $\mathrm{P} 2 \mathrm{Y}_{1}$ receptor mediates PLC activation via a $\mathrm{G}_{\alpha \mathrm{qq}}$ subunit and subsequently regulates intracellular calcium mobilization and platelet shape changes [Stalker et al., 2012]. P2 $Y_{12}$ receptor, on the other hand, is coupled to the $\mathrm{G}_{\alpha \mathrm{i}}$ subunit, which prevents the activation of adenylate cyclase, whereupon the intracellular cAMP concentration decreases. $\mathrm{P} 2 \mathrm{Y}_{12}$ receptor behaves as a negative regulator of platelet activation [Hechler and Gachet, 2011]. The P2Y $\mathrm{Y}_{12}$-dependent $\mathrm{G}_{\alpha \mathrm{i}}$ activation also potentiates the release of granule contents [Garcia et al., 2011] and can directly activate the $\alpha_{\mathrm{IIb}} \beta_{3}$ integrin via phosphoinositide-3 kinase [Jackson et al., 2004; Cosemans et al., 2006; Schoenwaelder et al., 2007; 
Gratacap et al ., 2011]. However in platelets stimulated by 2-AG the trend of adenylic nucleotides secretion [Signorello and Leoncini, 2014] does not parallel with the changes in cAMP intracellular concentration. For such reason ADP that could be secreted upon platelet activation by 2-AG cannot partecipate to the cAMP level reduction. In addition endocannabinoids inhibit platelet aggregation induced by several agonists such as ADP, thrombin or collagen [Levy et al., 1976; De Angelis et al., 2014]. cAMP is a control molecule of down-regulation of platelet activation [Smolenski, 2012; Raslan and Naseem, 2014]. Intracellular cAMP level reduction can be obtained by inhibiting adenylate cyclase or by stimulating cAMP-PDE. Since 2-AG does not affect adenylate cyclase, as shown in this report (Fig. 2), likely 2-AG acts on cAMP-PDE, by stimulating its activity. The cAMP-dependent PDE is a component of signalling pathway of platelets activation by 2-AG. In particular PDE3A seems to be involved in these mechanisms, as milrinone abolishes the 2-AG effect on cAMP. Platelets express at least three PDEs: cGMP-stimulated PDE2 and PDE5 and cGMP-inhibited PDE3A. PDE3A has a lower km for cAMP than PDE2 [Smolenski, 2012]. For these properties PDE3A exerts a greater influence on CAMP level in resting and activated platelets. Moreover PDE3A inhibition by milrinone (Fig. 1C) or cilostamide raises basal cAMP level and strongly inhibits platelet aggregation [Levy et al., 1976; Manns et al., 2002]. In contrast the PDE2 inhibitor EHNA has no effect on cAMP level (Fig. 1C) and platelet aggregation [De Angelis et al., 2014]. Here, we shows that 2-AG enhances PDE3A activity (Fig. 3A,B) producing a significant reduction of cAMP level. Previously it was shown that PDE3A activity is positively regulated by phosphorylation [Hunter et al., 2009; Zhang and Coleman, 2007]. In particular it has been demonstrated that thrombin stimulates PDE3A activity in human platelets through a phosphorylation mechanism which mainly may involve the PI3K/AKT signalling pathway [Zhang and Coleman, 2007]. In contrast, Hunter et al. [2009] have shown that in platelets stimulated by varying agonists, PDE3A is activated and phosphorylated on several serine residues (ser312, ser428, ser438, ser465, ser492), in parallel with the PKC substrate pleckstrin phosphorylation. According to the above cited authors [Hunter et al., 2009] phosphorylation and activation of PDE3A require PKC activation and is AKT-independent. In this report we show that in platelets stimulated with 2-AG PDE3A is phosphorylated (Fig. 4A,B). PDE3A phosphorylation parallels the PDE activation (Fig. 3A,B). Moreover PDE3A phosphorylation and activation are greatly inhibited by LY294002 and MK2206 suggesting that the PI3K/AKT pathway is involved in PDE3A phosphorylation and activation by 2-AG. Similarly, platelet modulators such as insulin [Lopez-Aparicio et al., 1993] and leptin [Elbatarny and Maurice, 2005] can stimulate phosphorylation and activation of PDE3A through the PI3K/AKT pathway. In addition in platelets stimulated by $2-\mathrm{AG}$ the PI3K/AKT pathway is actively involved in MLC phosphorylation and in some downstream events such as actin polymerization, ATP secretion and aggregation [Signorello and Leoncini, 2014]. Finally cAMP signalling regulates platelet MLC phosphorylation and shape change operating at the level of the RhoA/Rho kinaseMLC phosphatase signalling pathway [Aburima et al., 2013]. On the other hand data shown in this report obtained in 
platelets pretreated with the PLC or PKC inhibitor U73122 and GF109203X demonstrate that also the PLC/PKC pathway participates to the phosphorylation/activation of PDE3A induced by 2-AG as in platelets stimulated by 2 -AG PKC that is activated in platelets [Signorello et al., 2011b] directly phosphorylates PDE3A.

In conclusion in this report we provide evidence that AKT via the PI3K signalling pathway and PKC directly can phosphorylate/ activate PDE3A, producing an enhancement of cAMP hydrolysis. Likely 2-AG, stimulating pathways which lead to platelet activation and aggregation, strengthens its power through the reduction in cAMP intracellular levels.

\section{ACKNOWLEDGEMENT}

This work was supported by the Ministero della Ricerca Scientifica (Cofin MIUR 2009 project $\mathrm{n}^{\circ}$ 02030200605), Italy.

\section{REFERENCES}

Aburima A, Wraith KS, Raslan Z, Law R, Magwenzi S, Naseem KM. 2013. cAMP signaling regulates platelet myosin light chain (MLC) phosphorylation and shape change through targeting the RhoA-Rho kinase-MLC phosphatase signaling pathway. Blood. 122:3533-45.

Baldassarri S, Bertoni A, Bagarotti A, Sarasso C, Zanfa M, Catani MV, Avigliano L, Maccarrone M, Torti M, Sinigaglia F. 2008. The endocannabinoid 2-arachidonoylglycerol activates human platelets through non-CB1/CB2 receptors. J Thromb Haemost. 6:1772-9.

Born, GVR. 1962. Aggregation of blood platelets by adenosine diphosphate and its reversal. Nature, 194:927-9.

Brantl SA, Khandoga AL, Siess W. 2014. Activation of platelets by the endocannabinoids 2-arachidonoylglycerol and virodhamine is mediated by their conversion to arachidonic acid and thromboxane A2, not by activation of cannabinoid receptors. Platelets. 25:465-6.

Catani MV, Gasperi V, Catanzaro G, Baldassarri S, Bertoni A, Sinigaglia F, Avigliano L, Maccarrone M. 2010. Human platelets express authentic CB1 and CB2 receptors. Curr Neurovasc Res. 7:311-8.

Colman RW. 2004. Platelet cyclic adenosine monophosphate phosphodiesterases: targets for regulating plateletrelated thrombosis. Semin Thromb Hemost. 30:451-60. 
Cosemans JM, Munnix IC, Wetzker R, Heller R, Jackson SP, Heemskerk JW. 2006. Continuous signaling via PI3K isoforms beta and gamma is required for platelet ADP receptor function in dynamic thrombus stabilization. Blood 108:3045-52.

De Angelis V, Koekman AC, Weeterings C, Roest M, de Groot PG, Herczenik E, Maas C. 2014. Endocannabinoids control platelet activation and limit aggregate formation under flow. PLoS One. 9:e108282.

Derkinderen P, Ledent C, Parmentier M, Girault JA. 2001. Cannabinoids activate p38 mitogen-activated protein kinases through CB1 receptors in hippocampus. J Neurochem. 77:957-60.

Elbatarny HS, Maurice DH. 2005. Leptin-mediated activation of human platelets: involvement of a leptin receptor and phosphodiesterase 3A-containing cellular signaling complex. Am J Physiol Endocrinol Metab. 289:E695-702.

Gambaryan S, Kobsar A, Hartmann S, Birschmann I, Kuhlencordt PJ, Müller-Esterl W, Lohmann SM, Walter U. 2008. NO-synthase-/NO-independent regulation of human and murine platelet soluble guanylyl cyclase activity. J Thromb Haemost. 6:1376-84.

Garcia AE, Mada SR, Rico MC, Dela Cadena RA, Kunapuli SP. 2011. Clopidogrel, a P2Y12 Receptor Antagonist, Potentiates the Inflammatory Response in a Rat Model of Peptidoglycan Polysaccharide-Induced Arthritis. PLoS One 6:e26035.

Gratacap MP, Guillermet-Guibert J, Martin V, Chicanne G, Tronchere H, Gaits-Iacovoni F, Payrastre B. 2011. Regulation and roles of PI3Kbeta, a major actor in platelet signaling and functions. Adv Enzyme Regul. 51:106-16.

Haslam RJ, Dickinson NT, Jang EK. 1999. Cyclic nucleotides and phosphodiesterases in platelets. Thromb Haemost. 82:412-23.

Hechler B, Gachet C. 2011. P2 receptors and platelet function. Purinergic Signal. 7:9247-56.

Hunter RW, Mackintosh C, Hers I. 2009. Protein kinase C-mediated phosphorylation and activation of PDE3A regulate cAMP levels in human platelets. J Biol Chem. 284:12339-48.

Jackson SP, Yap CL, Anderson KE. 2004. Phosphoinositide 3-kinases and the regulation of platelet function. Biochem Soc Trans. 32:387-392. 
Jordà MA, Verbakel SE, Valk PJ, Vankan-Berkhoudt YV, Maccarrone M, Finazzi-Agrò A, Löwenberg B, Delwel R. 2002. Hematopoietic cells expressing the peripheral cannabinoid receptor migrate in response to the endocannabinoid 2-arachidonoylglycerol. Blood. 99: 2786-93.

Keown OP, Winterburn TJ, Wainwright CL, Macrury SM, Neilson I, Barrett F, Leslie SJ, Megson IL. $2010.2-$ arachidonyl glycerol activates platelets via conversion to arachidonic acid and not by direct activation of cannabinoid receptors. Br J Clin Pharmacol. 70:180-8.

Kishimoto S1, Gokoh M, Oka S, Muramatsu M, Kajiwara T, Waku K, Sugiura T. 2003. 2-arachidonoylglycerol induces the migration of HL-60 cells differentiated into macrophage-like cells and human peripheral blood monocytes through the cannabinoid CB2 receptor-dependent mechanism. J Biol Chem. 278:24469-75.

Kishimoto S, Kobayashi Y, Oka S, Gokoh M, Waku K, Sugiura T. 2004. 2-Arachidonoylglycerol, an endogenous cannabinoid receptor ligand, induces accelerated production of chemokines in HL-60 cells. J Biochem. 135:517-24.

Kobayashi Y, Arai S, Waku K, Sugiura T. 2001. Activation by 2-arachidonoylglycerol, an endogenous cannabinoid receptor ligand, of p42/44 mitogen-activated protein kinase in HL-60 cells. J Biochem. 129:665-9.

Lagarde M, Dechavanne M. 1977. Thrombin decreases platelet cyclic AMP in the absence of prostaglandin synthesis. Biomedicine. 27:110-2.

Leoncini G, Signorello MG, Bruzzese D, Di Braccio M, Grossi GC, Roma G. 2004. Mechanisms involved in the antiplatelet activity of 8-methyl-4-(1-piperazinyl)-7-(3-pyridinylmethoxy)-2H-1-benzopyran-2-one (RC414). Biochem Pharmacol. 67:911-8.

Levy R, Schurr A, Nathan I, Dvilanski A, Livne A. 1976. Impairment of ADP-induced platelet aggregation by hashish components. Thromb Haemost. 36:634-40.

López-Aparicio P, Belfrage P, Manganiello VC, Kono T, Degerman E. 1993. Stimulation by insulin of a serine kinase in human platelets that phosphorylates and activates the cGMP-inhibited cAMP phosphodiesterase. Biochem Biophys Res Commun. 193:1137-44.

Maccarrone M, Bari M, Menichelli A, Giuliani E, Del Principe D, Finazzi-Agrò A. Human platelets bind and degrade 2-arachidonoylglycerol, which activates these cells through a cannabinoid receptor. Eur J Biochem. 2001;268:819-25. 
Mach F, Montecucco F, Steffens S. 2009. Effect of blockage of the endocannabinoid system by CB(1) antagonism on cardiovascular risk. Pharmacol Rep. 61:13-21.

Malorni W, Bari M, Straface E, Battista N, Matarrese P, Finazzi-Agrò A, Del Principe D, Maccarrone M. 2004. Morphological evidence that 2-arachidonoylglycerol is a true agonist of human platelets. Thromb Haemost. 92:115961.

Manns JM, Brenna KJ, Colman RW, Sheth SB. 2002. Differential regulation of human platelet responses by cGMP inhibited and stimulated cAMP phosphodiesterases. Thromb Haemost. 87:873-9.

Petrosino S, Ligresti A, Di Marzo V. 2009. Endocannabinoid chemical biology: a tool for the development of novel therapies. Curr Opin Chem Biol. 13:309-20.

Randall MD. 2007. Endocannabinoids and the haematological system. Br J Pharmacol. 152:671-5.

Raslan Z, Naseem KM. 2014. The control of blood platelets by cAMP signalling. Biochem Soc Trans. 42:289-94.

Riba R, Oberprieler NG, Roberts W, Naseem KM. 2006. Von Willebrand factor activates endothelial nitric oxide synthase in blood platelets by a glycoprotein Ib-dependent mechanism. J Thromb Haemost. 4:2636-44.

Salzet M, Breton C, Bisogno T, Di Marzo V. 2000. Comparative biology of the endocannabinoid system possible role in the immune response. Eur J Biochem. 267:4917-27.

Schoenwaelder S, Ono A, Sturgeon S, Chan SM, Mangin P, Maxwell MJ, Turnbull S, Mulchandani M, Anderson K, Kauffenstein G, Rewcastle GW, Kendall J, Gachet C, Salem HH, Jackson SP. 2007. Identification of a unique cooperative phosphoinositide 3-kinase signaling mechanism regulating integrin alpha IIb beta 3 adhesive function in platelets. J Biol Chem. 282:28648-58.

Signorello MG, Giacobbe E, Leoncini G. 2011a. Activation by 2-arachidonoylglycerol of platelet p38MAPK/cPLA2 pathway. J Cell Biochem. 112:2794-802.

Signorello MG, Giacobbe E, Segantin A, Avigliano L, Sinigaglia F, Maccarrone M, Leoncini G. 2011b. Activation of human platelets by 2-arachidonoylglycerol: role of PKC in NO/cGMP pathway modulation. Curr Neurovasc Res. 8:200-9. 
Signorello MG, Giacobbe E, Leoncini G. 2013. The 2-arachidonoylglycerol effect on myosin light chain phosphorylation in human platelets. Biochimie. 95:162-8.

Signorello MG, Leoncini G. 2014. Effect of 2-arachidonoylglycerol on myosin light chain phosphorylation and platelet activation: The role of phosphatidylinositol 3 kinase/AKT pathway. Biochimie. 105:182-91.

Smolenski A. 2012. Novel roles of cAMP/cGMP-dependent signaling in platelets. J Thromb Haemost. 10:167-76.

Stalker TJ, Newman DK, Ma P, Wannemacher KM, Brass LF. 2012. Platelet signaling. Handb Exp Pharmacol. 210:59-85.

Sugiura T, Kodaka T, Nakane S, Kishimoto S, Kondo S, Waku K. 1988. Detection of an endogenous cannabimimetic molecule, 2-arachidonoylglycerol, and cannabinoid CB1 receptor mRNA in human vascular cells: is 2arachidonoylglycerol a possible vasomodulator? Biochem Biophys Res Commun. 243:838-43.

Sugiura T, Kodaka T, Kondo S, Tonegawa T, Nakane S, Kishimoto S, Yamashita A, Waku K. 1997. Inhibition by 2arachidonoylglycerol, a novel type of possible neuromodulator, of the depolarization-induced increase in intracellular free calcium in neuroblastoma x glioma hybrid NG108-15 cells. Biochem Biophys Res Commun. 233:207-10.

Sugiura T. Waku K. 2002. Cannabinoid receptors and their endogenous ligands. J Biochem. 132:7-12.

Sugiura T, Kishimoto S, Oka S, Gokoh M. Biochemistry, pharmacology and physiology of 2-arachidonoylglycerol, an endogenous cannabinoid receptor ligand. 2006. Prog Lipid Res. 45:405-46.

Zhang W, Colman RW. 2007. Thrombin regulates intracellular cyclic AMP concentration in human platelets through phosphorylation/activation of phosphodiesterase 3A. Blood. 110:1475-82.

Zhang G, Xiang B, Dong A, Skoda RC, Daugherty A, Smyth SS, Du X, Li Z. 2011. Biphasic roles for soluble guanylyl cyclase (sGC) in platelet activation. Blood. 118:3670-9. 


\section{LEGENDS}

Fig. 1. Effect of 2-AG on cAMP level. In panel $\mathbf{A}$ washed platelets $\left(1.0 \times 10^{9}\right.$ platelets $\left./ \mathrm{mL}\right)$, prewarmed at $37^{\circ} \mathrm{C}$, were stimulated for $120 \mathrm{sec}$ with varying concentrations of 2-AG. In panel B washed platelets $\left(1.0 \times 10^{9}\right.$ platelets $\left./ \mathrm{mL}\right)$ were stimulated with $10 \mu \mathrm{M} 2-\mathrm{AG}$ and incubated at $37^{\circ}$ as indicated. In panel $\mathbf{C}$ washed platelets $\left(1.0 \times 10^{9}\right.$ platelets $\left./ \mathrm{mL}\right)$ were preincubated at $37^{\circ} \mathrm{C}$ for 10 min with saline or $50 \mu \mathrm{M}$ SQ22536 (S), $50 \mu \mathrm{M}$ EHNA (E), $10 \mu \mathrm{M}$ milrinone (M), $20 \mu \mathrm{M}$ LY294002 (L), $10 \mu \mathrm{M}$ MK2206 (MK), $10 \mu \mathrm{M}$ U73122 (U), $1 \mu \mathrm{M}$ SQ29548 (SQ) and $100 \mu \mathrm{M}$ ASA (ASA) and then stimulated for $120 \mathrm{sec}$ with $10 \mu \mathrm{M}$ 2-AG. At the end of incubation suitable aliquots were analyzed for cAMP content by EIA kit as reported in Methods. Data are the mean \pm SD of four independent experiments carried out in duplicate. One way ANOVA-Bonferroni's post hoc test: ${ }^{*} \mathrm{p}<0.01, \# \mathrm{p}<0.05$. Student's t-test: ${ }^{*} \mathrm{p}<0.0005$ vs none; ${ }^{* *} \mathrm{p}<0.0001$, $\# \mathrm{p}<0.0005, \mathrm{p}<0.01$ vs $2-\mathrm{AG}$.

Fig. 2. Effect of 2-AG on adenylate cyclase activity. Washed platelets $\left(1.0 \times 10^{9}\right.$ platelets $\left./ \mathrm{mL}\right)$, prewarmed at $37^{\circ} \mathrm{C}$ for 10 min with saline, $100 \mu \mathrm{M}$ IBMX or $20 \mu \mathrm{M}$ forskolin $(\mathbf{F})$, were stimulated for $120 \mathrm{sec}$ with varying concentrations of 2-AG as indicated. At the end of incubation suitable aliquots were analyzed for adenylate cyclase activity by EIA kit as reported in Methods. Data are the mean \pm SD of four independent experiments carried out in duplicate. Student's t-test: $* \mathrm{p}<0.0001$ vs none.

Fig. 3. Effect of 2-AG on PDE activity. In panel A washed platelets $\left(1.0 \times 10^{9}\right.$ platelets $\left./ \mathrm{mL}\right)$, prewarmed at $37^{\circ} \mathrm{C}$, were stimulated for $120 \mathrm{sec}$ with varying concentrations of 2-AG. In panel B washed platelets $\left(1.0 \times 10^{9}\right.$ platelets $\left./ \mathrm{mL}\right)$, prewarmed at $37^{\circ} \mathrm{C}$, were stimulated with $10 \mu \mathrm{M}$ 2-AG. In panel $\mathbf{C}$ washed platelets $\left(1.0 \times 10^{9}\right.$ platelets $\left./ \mathrm{mL}\right)$ were preincubated at $37^{\circ} \mathrm{C}$ for $10 \mathrm{~min}$ with saline or $50 \mu \mathrm{M}$ SQ22536 (S), $50 \mu \mathrm{M}$ EHNA (E), $10 \mu \mathrm{M}$ milrinone (M), $20 \mu \mathrm{M}$ LY294002 (L), $10 \mu \mathrm{M}$ MK2206 (MK), $10 \mu \mathrm{M}$ U73122 (U), $1 \mu \mathrm{M}$ SQ29548 (SQ) and $100 \mu \mathrm{M}$ ASA (ASA) and then stimulated for $120 \mathrm{sec}$ with $10 \mu \mathrm{M}$ 2-AG. At the end of incubation suitable aliquots were analyzed for PDE activity by EIA kit as reported in Methods. Data are the mean \pm SD of four independent experiments carried out in duplicate. One way ANOVA-Bonferroni's post hoc test: ${ }^{*} \mathrm{p}<0.01$, $\# \mathrm{p}<0.05$. Student's t-test: ${ }^{*} \mathrm{p}<0.0005$ vs none; $* * \mathrm{p}<0.0001$, $\# \mathrm{p}<0.0005, \mathrm{p}<0.01$ vs $2-\mathrm{AG}$.

Fig. 4. Phosphorylation of PDE3A in platelets stimulated with 2-AG. In panel A washed platelets $\left(1.0 \times 10^{9}\right.$ platelets $/ \mathrm{mL}$ ), prewarmed at $37^{\circ} \mathrm{C}$, were incubated for $120 \mathrm{sec}$ with varying concentrations of 2-AG. In panel $\mathbf{B}$ washed platelets $\left(1.0 \times 10^{9}\right.$ platelets $\left./ \mathrm{mL}\right)$, prewarmed at $37^{\circ} \mathrm{C}$, were stimulated with $10 \mu \mathrm{M} 2$-AG. At the end of incubation suitable aliquots were immunoprecipitated with anti-p-PDE3A and immunoblotted with anti-phosphoserine (p-Ser) as detailed in Methods. Blots are representative of four independent experiments. In the lower panels densitometric scanning \pm SD of PDE3A phosphorylation of four experiments is shown. One way ANOVA-Bonferroni's post hoc test: $\# \mathrm{p}<0.01 ; * \mathrm{p}<0.001$. 
Fig. 5. Effect of varying inhibitors on phosphorylation of PDE3A induced by $2-\mathrm{AG}$. Washed platelets $\left(1.0 \times 10^{9}\right.$ platelets $/ \mathrm{mL}$ ) were preincubated at $37^{\circ} \mathrm{C}$ for $10 \mathrm{~min}$ with saline or $1 \mu \mathrm{M}$ SQ29548 (SQ), $100 \mu \mathrm{M}$ aspirin $(\mathbf{A S A}), 10 \mu \mathrm{M}$ milrinone (M), $50 \mu \mathrm{M}$ EHNA (E), $10 \mu \mathrm{M}$ GF109203X (G), $10 \mu \mathrm{M}$ U73122 (U), $20 \mu \mathrm{M}$ LY294002 (L) and $10 \mu \mathrm{M}$ MK2206 (MK), and then stimulated for $120 \mathrm{sec}$ with $10 \mu \mathrm{M}$ 2-AG. At the end of incubation suitable aliquots were immunoprecipitated with anti-p-PDE3A and immunoblotted with anti-phosphoserine (p-Ser) as detailed in Methods. Blots are representative of four independent experiments. In the lower panel densitometric scanning \pm SD of PDE3A phosphorylation of four experiments is reported. Student's t-test: *p<0.0001 vs none; \#p<0.0001 vs 2-AG

Fig. 6. Phosphorylation of PDE3A (ser290/293) during 2-AG activation of platelets. In panel A washed platelets $\left(1.0 \times 10^{9}\right.$ platelets $\left./ \mathrm{mL}\right)$, prewarmed at $37^{\circ} \mathrm{C}$, were incubated for $120 \mathrm{sec}$ with varying concentrations of $2-\mathrm{AG}$. In panel B washed platelets $\left(1.0 \times 10^{9}\right.$ platelets $\left./ \mathrm{mL}\right)$, prewarmed at $37^{\circ} \mathrm{C}$, were stimulated with $10 \mu \mathrm{M} 2-\mathrm{AG}$. At the end of incubation suitable aliquots were immunoblotted with anti-p-PDE3A (ser290/293) as detailed in Methods. Blots are representative of four independent experiments. In the lower panels densitometric scanning \pm SD of PDE3A phosphorylation of four experiments is reported. One way ANOVA-Bonferroni's post hoc test: $\# \mathrm{p}<0.01 ;{ }^{*} \mathrm{p}<0.001$.

Fig. 7. Effect of varying inhibitors on phosphorylation of PDE3A (ser290/293) induced by 2-AG. Washed platelets $\left(1.0 \times 10^{9}\right.$ platelets $\left./ \mathrm{mL}\right)$ were preincubated at $37^{\circ} \mathrm{C}$ for 10 min with saline or $50 \mu \mathrm{M}$ EHNA $(\mathbf{E}), 10 \mu \mathrm{M}$ milrinone $(\mathbf{M})$, $20 \mu \mathrm{M}$ LY294002 (L) or $10 \mu \mathrm{M}$ MK2206 (MK) and then stimulated for $120 \mathrm{sec}$ with $10 \mu \mathrm{M}$ 2-AG. At the end of incubation suitable aliquots were immunoblotted with anti-p-PDE3A (ser290/293) as detailed in Methods. Blots are representative of four independent experiments. In the lower panel densitometric scanning \pm SD of PDE3A phosphorylation of four experiments is shown. Student's t-test: *p<0.0001 vs none; \#p<0.0001 vs 2-AG 